\title{
Semidiscrete Least-Squares Methods for Second Order Parabolic Problems With Nonhomogenous Data
}

\author{
By J. Thomas King
}

\begin{abstract}
Recently, Bramble and Thomée proposed semidiscrete least-squares methods for the heat equation. In this paper we extend these methods to variable coefficient parabolic operators with nonhomogeneous equations and boundary conditions.
\end{abstract}

1. Introduction. Recently there has been much interest in variational methods for approximating the solution of parabolic problems (cf. [8], [12], [14]).

For essential boundary conditions, the author proposed weighted least-squares methods for parabolic problems [10]. These methods are based on the ideas of Bramble and Schatz [3]. Another way of implementing the ideas of [3] to parabolic problems has been proposed by Bramble and Thomée [6]. In [6] the authors give semidiscrete least-squares methods for the heat equation under homogeneous boundary conditions.

The purpose of this paper is to generalize the methods of [6] to variable coefficient second order parabolic operators with nonhomogeneous data. The error analysis given here will be similar to that of [6], however we need to significantly change some details.

2. The Initial-Boundary Value Problem. Let $\Omega$ be a bounded open domain in Euclidean $N$-space with boundary $\partial \Omega$, of class $C^{\infty}$ (cf. [1]). For $0<T<\infty$ we put $Q=\Omega \times(0, T]$ and $\Gamma=\partial \Omega \times(0, T]$.

We shall consider, in $Q$, the parabolic operator $L$ defined by

$$
L u=A(x, t) u-u_{t}=\sum_{i, i=1}^{N} D_{x_{i}}\left(a_{i i}(x, t) D_{x_{i}} u\right)-u_{t}
$$

and the following initial-boundary value problem:

$$
L u=f \quad \text { in } Q, \quad u=g \text { on } \Gamma, \quad u(\cdot, 0)=u_{0} \quad \text { in } \Omega
$$

where $f, g, u_{0}$ are given. We assume that $A$ is uniformly elliptic in the closure of $Q$, $Q$, and for convenience assume that the coefficients $a_{i j}$ are in $C^{\infty}(\bar{Q})$.

We will need the following function spaces. For $k \geqq 0$, an integer, $H^{k}(\Omega)$ will denote the usual Sobolev space of order $k$ on $\Omega$ with norm

$$
\|\phi\|_{k}=\left[\sum_{|\alpha| \leq k}\left\|D^{\alpha} \phi\right\|^{2}\right]^{1 / 2}
$$

where $\|\phi\|=\left(\int_{\Omega}|\phi(x)|^{2} d x\right)^{1 / 2}$.

Received August 9, 1971, revised March 13, 1972, November 17, 1972, February 20, 1973. AMS (MOS) subject classifications (1970). Primary 65N99; Secondary 49D25, 35K 20.

Key words and phrases. Semidiscrete least-squares method, error analysis, parabolic equation.

Copyright (c) 1974, American Mathematical Society 
We will denote the corresponding inner product on $H^{0}(\Omega)=L_{2}(\Omega)$ by $\langle\langle\cdot, \cdot\rangle\rangle$. The inner product and norm on $L_{2}(\partial \Omega)$ will be denoted by $\langle\cdot, \cdot\rangle$ and $|\cdot|$ respectively.

For the semidiscrete methods which follow we will need to introduce finitedimensional subspaces of $H^{2}(\Omega)$ having certain approximation theoretic properties depending on a small parameter $h$. Specifically we will assume throughout this paper that $S_{h}{ }^{r}$ is any finite-dimensional subspace of $H^{2}(\Omega)$ having the following property:

$\left(^{*}\right)$ For any $u \in H^{*}(\Omega)$ with $2 \leqq s \leqq r$ there exists a constant $C$, independent of $h$ and $u$, such that

$$
\inf _{\phi \in S_{\Lambda^{\prime}}} \sum_{i=0}^{2} h^{i-2}\|u-\phi\|_{i} \leqq C h^{s-2}\|u\|_{s} .
$$

Examples of such subspaces are contained in [2], [7], [9], and [13].

3. Implicit Semidiscrete Methods. In this section we give semidiscrete leastsquares schemes for finding approximate solutions of the initial-boundary value problem using the subspaces $S_{h}{ }^{r}$. The schemes proposed here are based on the classical implicit finite-difference approximation for $L$.

Before making these ideas more precise we will need some notation. Let $k=$ $T / M$ where $M$ is a positive integer and let $t_{n}=n k$ for $n=0, \cdots, M$. We will use the notation $u^{n}=u\left(\cdot, t_{n}\right)$ and $A_{n}=A\left(\cdot, t_{n}\right)$.

We shall approximate the solution of problem (2.1) by a function of the form

$$
v(x, t)=\sum_{i=1}^{\nu} c_{i}(t) \phi_{i}(x)
$$

where $\left\{\phi_{i}\right\}_{i=1}^{*}$ is a suitable local basis for $S_{h}{ }^{r}$ and $c_{i}$ is defined on the mesh: $0<$ $t_{1}<\cdots<t_{M-1}<T$.

The implicit approximation schemes are defined as follows:

For given $S_{h}{ }^{r}$ having local basis $\left\{\phi_{i}\right\}_{i=1}^{0}$ and given $\gamma$ satisfying $0 \leqq \gamma \leqq \frac{3}{2}$, find a function $v$ of the form (3.1) such that

$$
\begin{gathered}
\text { (i) }\left\langle\left\langle u_{0}-v^{0}, \phi\right\rangle\right\rangle=0 \text { for all } \phi \in S_{h}^{r} \text { and for } n=0, \cdots, M-1, \\
\text { (ii) }\left\langle\left\langle k f^{n+1}-v^{n}-\left(k A_{n+1}-1\right) v^{n+1},\left(k A_{n+1}-1\right) \phi\right\rangle\right\rangle \\
+k^{2} h^{-2 \gamma}\left\langle g^{n+1}-v^{n+1}, \phi\right\rangle=0 \text { for all } \phi \in S_{h}^{r} .
\end{gathered}
$$

We remark that the elements of $S_{h}{ }^{r}$ are not required to satisfy boundary conditions, and only $L_{2}$ inner products are involved in the computation of $v$. The solution of (3.2), for each $n$, is determined by solving a linear system of algebraic equations whose coefficients depend only on $F=\left\{f, g, u_{0}\right\}, h, k$, and $\gamma$. The linear system yields a symmetric matrix which is sparse if we take $S_{h}{ }^{r}$ to be a certain class of splines with a suitable local basis. Note also that we could choose $v^{0} \equiv u_{0}$ in place of (i) in (3.2).

For notational simplicity it is convenient to introduce the following definitions. For $u \in H^{2}(\Omega)$ we define the operator $L_{k}{ }^{n} u \equiv\left(k A_{n}-1\right) u$.

Let $\Lambda$ be the product space $H^{0}(\Omega) \times H^{0}(\partial \Omega)$ with inner product

$$
[U, V]=\langle\langle f, \tilde{f}\rangle\rangle+k^{2} h^{-2 \gamma}\langle g, \tilde{g}\rangle
$$

where $U=\{f, g\}$ and $V=\{\tilde{f}, \tilde{g}\}$. We put $\|U\|_{\Lambda}=[U, U]^{1 / 2}$.

Let ${T_{k}}^{n}$ be the map $\left\{L_{k}{ }^{n} u,\left.u\right|_{\partial \Omega}\right\}$ from $H^{2}(\Omega)$ into $\Lambda$. Then $\left\|T_{k}{ }^{n} u\right\|_{\Lambda}$ is a norm on $H^{2}(\Omega)$. 
Existence. We assume that $u_{0} \in H^{0}(\Omega), f^{n} \in H^{0}(\Omega)$, and $g^{n} \in H^{0}(\partial \Omega)$ for each $n=1, \cdots, M$. Since the matrix $\left\{\left\langle\left\langle\phi_{i}, \phi_{i}\right\rangle\right\rangle\right\}$ is positive definite, (i) has a unique solution $\left\{c_{1}{ }^{0}, \cdots, c_{v}{ }^{0}\right\}$. Now suppose that $\left\{c_{1}{ }^{n}, \cdots, c_{\nu}{ }^{n}\right\}$ have been found; then (ii) has a unique solution if and only if the only solution of the system

$$
\left[T_{k}^{n+1} v^{n+1}, T_{k}^{n+1} \phi_{i}\right]=0, \quad 1 \leqq j \leqq \nu,
$$

is the trivial solution. Multiplying (3.3) by $c_{i}{ }^{n+1}$ and summing over $j$ yields

$$
\left\|T_{k}^{n+1} v^{n+1}\right\|_{\Lambda}^{2}=0
$$

so that $L_{k}{ }^{n+1} v^{n+1}=0$ in $\Omega$ and $v^{n+1}=0$ on $\partial \Omega$. Hence $v^{n+1} \equiv 0$.

Error Analysis. We will need the following a priori inequality for the operator $L_{k}{ }^{n}$ which is essentially proved in [6].

LEMMA 3.1. There exists a constant $\alpha \geqq 1$ such that, for $u \in H^{2}(\Omega)$ and $1 \leqq n \leqq M$,

$$
\|u\|^{2} \leqq\left\|L_{k}^{n} u\right\|^{2}+\alpha k^{1 / 2}|u|^{2} .
$$

We will also make use of the following lemma which is essentially proved in [6].

LEMMA 3.2. Suppose $u \in H^{*}(\Omega)$ with $2 \leqq s \leqq r$ and $C_{0}>0$. If $k \geqq C_{0} h^{4 \gamma / 3}$ $\left(0 \leqq \gamma \leqq \frac{3}{2}\right)$ then

$$
\inf _{\phi \in S_{\Lambda^{r}}}\left\|T_{k}^{n}(u-\phi)\right\|_{\Lambda} \leqq C k h^{\theta-2}\|u\|_{。}
$$

where $C$ is independent of $h, k$, and $u$.

We will also make use of the following easily proved estimate.

LEMMA 3.3. Suppose $a_{n}, b_{n}$, and $c_{n}$ are nonnegative. If $\sigma \geqq 0$, and, for $n=0$, $1, \cdots, M-1$,

$$
a_{n+1}^{2} \leqq b_{n+1}^{2}+\left(1+\frac{\sigma}{M}\right) a_{n}^{2}+c_{n+1}^{2}+2\left(1+\frac{\sigma}{M}\right)^{1 / 2} a_{n} c_{n+1}
$$

then there exists a constant $C$, independent of $M$, such that

$$
\max _{0 \leq n \leq M} a_{n} \leqq C\left\{M^{1 / 2} \max _{0 \leq n \leq M} b_{n}+M \max _{0 \leq n \leq M} c_{n}+a_{0}\right\}
$$

We are now in a position to analyze the error in scheme (3.2). Let $u$ be the solution of (2.1), $v$ be the solution of (3.2), and put $e=u-v$.

THEOREM 3.1. If $u(\cdot, t) \in H^{*}(\Omega)$ for each $t \in(0, T]$ with $2 \leqq s \leqq r$ and $u \in$ $C^{2}(\bar{Q})$, then, for $k \geqq \alpha^{2 / 3} h^{4 \gamma / 3}$, we have

$$
\max _{0 \leq n \leq M}\left\|e^{n}\right\| \leqq C\left\{h^{2}\left\|u_{0}\right\|_{2}+k^{1 / 2} h^{s-2} \max _{1 \leq n \leq M}\left\|u^{n}\right\|_{s}+k \max _{t \in[0, T]}\left\|D_{t}^{2} u(\cdot, t)\right\|\right\} .
$$

We remark that $\alpha$ is the constant of Lemma 3.1.

Proof. We have from (3.4) that

$$
\left\|e^{n+1}\right\|^{2} \leqq\left\|L_{k}^{n+1} e^{n+1}\right\|^{2}+\alpha k^{1 / 2}\left|e^{n+1}\right|^{2}
$$

So that, for $k \geqq \alpha^{2 / 3} h^{4 \gamma / 3}$,

$$
\left\|e^{n+1}\right\|^{2} \leqq\left\|T_{k}^{n+1} e^{n+1}\right\|_{\Lambda}^{2}=\left[T_{k}^{n+1} e^{n+1}, T_{k}^{n+1} u^{n+1}\right]-\left[T_{k}^{n+1} e^{n+1}, T_{k}^{n+1} v^{n+1}\right] .
$$

Now let $\psi^{n+1} \in S_{h}{ }^{r}$ be such that

$$
\left[T_{k}^{n+1}\left(u^{n+1}-\psi^{n+1}\right), T_{k}^{n+1} \phi\right]=0 \quad \text { for all } \phi \in S_{h}^{r} .
$$


By an elementary application of Taylor's theorem we have

$$
L_{k}^{n+1} u^{n+1}=k f^{n+1}-u^{n}+\rho^{n+1}
$$

where $\left\|\rho^{n+1}\right\| \leqq C k^{2} \max _{t \in[0, T]}\left\|D_{t}{ }^{2} u(\cdot, t)\right\|$.

We now use (3.2) and (3.9) in (3.7) to yield

$$
\left\|e^{n+1}\right\|^{2} \leqq\left\langle\left\langle e^{n}-\rho^{n+1}, L_{k}^{n+1}\left(v^{n+1}-\psi^{n+1}\right)\right\rangle\right\rangle+\left[T_{k}^{n+1} e^{n+1}, T_{k}^{n+1}\left(u^{n+1}-\psi^{n+1}\right)\right] .
$$

By virtue of (3.10) and the definition of $\psi^{n+1}$ we then have

$$
\left\|e^{n+1}\right\|^{2} \leqq\left\langle\left\langle e^{n}-\rho^{n+1}, L_{k}^{n+1}\left(v^{n+1}-\psi^{n+1}\right)\right\rangle\right\rangle+\left\|T_{k}^{n+1}\left(u^{n+1}-\psi^{n+1}\right)\right\|_{\Lambda}^{2} .
$$

We now estimate the first term on the right-hand side of (3.11). We subtract (3.8) from (3.2) and use the relation (3.9) to yield

$$
\left[T_{k}^{n+1}\left(v^{n+1}-\psi^{n+1}\right), T_{k}^{n+1} \phi\right]=\left\langle\left\langle e^{n}-\rho^{n+1}, L_{k}^{n+1} \phi\right\rangle\right\rangle
$$

where $\phi \in S_{h}{ }^{r}$ is arbitrary. Choosing $\phi=v^{n+1}-\psi^{n+1}$ in (3.12) implies that

$$
\left\|L_{k}^{n+1}\left(v^{n+1}-\psi^{n+1}\right)\right\|^{2} \leqq\left|\left\langle\left\langle e^{n}-\rho^{n+1}, L_{k}^{n+1}\left(v^{n+1}-\psi^{n+1}\right)\right\rangle\right\rangle\right|
$$

so that, by the Schwarz inequality,

$$
\left\|L_{k}^{n+1}\left(v^{n+1}-\psi^{n+1}\right)\right\| \leqq\left\|e^{n}-\rho^{n+1}\right\| .
$$

Using the Schwarz inequality and (3.13) in (3.11) yields

$$
\left\|e^{n+1}\right\|^{2} \leqq\left\|T_{k}^{n+1}\left(u^{n+1}-\psi^{n+1}\right)\right\|_{\Lambda}^{2}+\left\|e^{n}-\rho^{n+1}\right\|^{2}
$$

so that

$$
\left\|e^{n+1}\right\|^{2} \leqq\left\|T_{k}^{n+1}\left(u^{n+1}-\psi^{n+1}\right)\right\|_{\Lambda}^{2}+\left\|e^{n}\right\|^{2}+\left\|\rho^{n+1}\right\|^{2}+2\left\|e^{n}\right\|\left\|\rho^{n-1}\right\| .
$$

It follows from (3.15) by an application of Lemma 3.3 with $\sigma=0$ that

$$
\begin{aligned}
\max _{1 \leqq n \leqq M}\left\|e^{n}\right\| \leqq C & \left\{k^{-1 / 2} \max _{1 \leq n \leqq M}\left\|T_{k}^{n}\left(u^{n}-\psi^{n}\right)\right\|_{\Lambda}\right. \\
& \left.+\left\|u_{0}-v^{0}\right\|+k^{-1} \max _{1 \leq n \leqq M}\left\|\rho^{n}\right\|\right\} .
\end{aligned}
$$

The result (3.6) follows from (3.16) by an application of Lemma 3.2 and property (*).

4. Crank-Nicolson Semidiscrete Methods. The implicit schemes of the previous section have, at best, order of convergence $O(k)$. In order to improve the order of convergence it is necessary to discretize the time variable with higher order accuracy. The schemes given here are based on a Crank-Nicolson type finite-difference approximation for the operator $L$. It will be shown that the order of convergence can be improved to $O\left(k^{2}\right)$.

We will use the notation $u^{n+1 / 2}=\frac{1}{2}\left(u^{n}+u^{n+1}\right)$. We shall again approximate the solution of problem (2.1) by a function of the form (3.1); however, we will now take $v^{0} \equiv u_{0}$. We will use the notation $L_{k}{ }^{n} u=\left(k A_{n}+1\right) u$.

The approximation schemes are defined as follows:

For a given $S_{h}{ }^{r}$ having local basis $\left\{\phi_{i}\right\}_{i=1}{ }^{2}$ and given $\gamma$ satisfying $0 \leqq \gamma \leqq \frac{3}{2}$, find a function of the form (3.1), with $u_{0} \equiv v^{0}$, such that, for $n=0, \cdots, M-1$, 


$$
\left\langle\left\langle k f^{n+1 / 2}-\tilde{L}_{k / 2}^{n} v^{n}-L_{k / 2}^{n+1} v^{n+1}, L_{k / 2}^{n+1} \phi\right\rangle\right\rangle+k^{2} h^{-2 \gamma}\left\langle g^{n+1}-v^{n+1}, \phi\right\rangle=0
$$

for all $\phi \in S_{h}{ }^{r}$.

Error Analysis. We will again need an a priori inequality for the operator $L_{k / 2}{ }^{n}$ which is essentially proved in [6].

LEMMA 4.1. There is a constant $\beta \geqq 1$ such that, for $u \in H^{2}(\Omega)$ and $1 \leqq n \leqq M$,

$$
\left\|\tilde{L}_{k / 2}^{n} u\right\|^{2} \leqq(1+k)\left\{\left\|L_{k / 2}^{n} u\right\|^{2}+\beta|u|^{2}\right\} .
$$

Let $u$ be the solution of (2.1), $v$ be the solution of the Crank-Nicolson scheme (4.1), and put $e=u-v$.

THEOREM 4.1. If $u(\cdot, t) \in H^{s}(\Omega)$ for each $t \in(0, T]$ with $2 \leqq s \leqq r$ and $u \in$ $C^{3}(Q)$ then for $k \geqq C_{0} h^{\gamma}$, with $C_{0} \geqq \max \left\{\alpha^{2 / 3}, \beta\right\}$ we have

$$
\max _{1 \leqq n \leqq M}\left\|e^{n}\right\| \leqq C\left\{k^{1 / 2} h^{s-2} \max _{1 \leqq n \leqq M}\left\|u^{n}\right\|_{s}+k^{2} \max _{t \in[0, T]}\left\|D_{t}^{3} u(\cdot, t)\right\|\right\}
$$

where $\alpha$ and $\beta$ are the constants of Lemmas 3.1 and 4.1 respectively and $C$ is independent of $h, k$, and $u$.

Note that we have assumed the more restrictive condition $k \geqq C_{0} h^{\gamma}$.

Proof. As in the proof of Theorem 3.1 we have

$$
\left\|e^{n+1}\right\|^{2} \leqq\left\|T_{k / 2}^{n+1} e^{n+1}\right\|_{\Lambda}^{2} \text {. }
$$

Using the relation

$$
L_{k / 2}^{n+1} u^{n+1}=k f^{n+1 / 2}-L_{k / 2}^{n} u^{n}+\rho^{n+1 / 2}
$$

where

$$
\left\|\rho^{n+1 / 2}\right\| \leqq C k^{3} \max _{t \in[0, T]}\left\|D_{t}^{3} u(\cdot, t)\right\|
$$

and arguments analogous to those in the proof of Theorem 3.1 it can be shown that the right-hand side of $(4.4)$ is bounded by

$$
\left\|T_{k / 2}^{n+1}\left(u^{n+1}-\psi^{n+1}\right)\right\|^{2}+\left|\left\langle\left\langle\tilde{L}_{k / 2}^{n} e^{n}-\rho^{n+1 / 2}, L_{k / 2}^{n+1}\left(v^{n+1}-\psi^{n+1}\right)\right\rangle\right\rangle\right|
$$

where $\psi^{n+1} \in S_{h}{ }^{r}$ is defined such that

$$
\left[T_{k / 2}^{n+1}\left(u^{n+1}-\psi^{n+1}\right), T_{k / 2}^{n+1} \phi\right]=0 \quad \text { for all } \phi \in S_{h}^{r} .
$$

We estimate the last term in (4.6) by methods analogous to those used in the proof of Theorem 3.1. We subtract (4.7) from (4.1) and use the relation (4.5) to yield

$$
\left[T_{k / 2}^{n+1}\left(v^{n+1}-\psi^{n+1}\right), T_{k / 2}^{n+1} \phi\right]=\left\langle\left\langle\tilde{L}_{k / 2}^{n} e^{n}-\rho^{n+1 / 2}, L_{k / 2}^{n+1} \phi\right\rangle\right\rangle
$$

where $\phi \in S_{h}{ }^{r}$ is arbitrary. Choosing $\phi=v^{n+1}-\psi^{n+1}$ in (4.8) implies that

$$
\left\|L_{k / 2}^{n+1}\left(v^{n+1}-\psi^{n+1}\right)\right\| \leqq\left\|\tilde{L}_{k / 2}^{n} e^{n}-\rho^{n+1 / 2}\right\| .
$$

Hence, from (4.4) through (4.9), we conclude that, for $0 \leqq n \leqq M-1$,

$$
\left\|e^{n+1}\right\|^{2} \leqq\left\|T_{k / 2}^{n+1} e^{n+1}\right\|_{\Lambda}^{2} \leqq\left\|T_{k / 2}^{n+1}\left(u^{n+1}-\psi^{n+1}\right)\right\|_{\Lambda}^{2}+\left\|L_{k / 2}^{n} e^{n}-\rho^{n+1 / 2}\right\|^{2} .
$$

Hence from the assumption $k \geqq C_{0} h^{\gamma}$ and Lemma 4.1 it follows that 


$$
\left\|e^{n+1} \mid\right\|^{2} \leqq\left\|T_{k / 2}^{n+1} e^{n+1}\right\|_{\Lambda}^{2} \leqq\left\|T_{k / 2}^{n+1}\left(u^{n+1}-\psi^{n+1}\right)\right\|_{\Lambda}^{2}
$$

$$
+(1+k)\left\|T_{k / 2}^{n} e^{n}\right\|_{\Lambda}^{2}+\left\|\rho^{n+1 / 2}\right\|^{2}+2(1+k)^{1 / 2}\left\|\rho^{n+1 / 2}\right\|\left\|T_{k / 2}^{n} e^{n}\right\|_{\Lambda} .
$$

An application of Lemma 3.3 with $\sigma=T$ in (4.11) yields

$$
\max _{1 \leq n \leq M}\left\|T_{k / 2}^{n} e^{n}\right\|_{\Lambda} \leqq C\left\{k^{-1 / 2} \max _{1 \leq n \leq M}\left\|T_{k / 2}^{n}\left(u^{n}-\psi^{n}\right)\right\|_{\Lambda}+k^{-1} \max _{1 \leq n \leq M}\left\|\rho^{n-1 / 2}\right\|\right\}
$$

from which (4.3) easily follows by an application of Lemma 3.2 and observing that (4.4) is valid for any $n=0, \cdots, M-1$.

Discussion of Results. The bounds in the error estimates (3.6) and (4.3) involve certain norms of the solution $u$ of problem (2.1). Under slightly stronger regularity assumptions on $u$ (i.e., the data $F=\left\{f, g, u_{0}\right\}$ must be sufficiently regular) these norms on $u$ can be bounded by certain Hölder norms on the data (cf. Theorem 5.2 on p. 320 of [11]).

For the purely implicit scheme (3.2) suppose we take $r=4$ and $S_{h}{ }^{4}$ to be cubic splines or the Hermite space of piecewise cubic polynomials ([4], [5], [9]). Then if

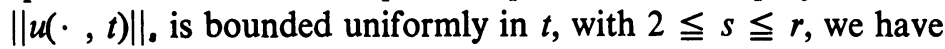

$$
\max _{0 \leq n \leq M}\left\|e^{n}\right\|=O\left(k^{1 / 2} h^{s-2}+k\right) .
$$

Hence, since $k \geqq \alpha^{2 / 3} h^{4} \gamma / 3$, we have that the error is of order $O(k)$ for $s=4$ and $\gamma=\frac{3}{2}$ or for $s=3$ and $\gamma=\frac{3}{2}$.

For the Crank-Nicolson scheme (4.1) we find that the error is $O\left(k^{2}\right)$ if $\gamma=\frac{4}{3}$ and $\|u(\cdot, t)\|_{4}$ is bounded uniformly in $t$.

The Crank-Nicolson methods of [8] yield error estimates of the form

$$
\max _{0 \leq n \leq M}\left\|e^{n}\right\|=O\left(h^{2 s-1}+k^{2}\right)
$$

where the solution of (2.1) satisfies $u(\cdot, t) \in H^{2 s}$ and the space of approximants consists of piecewise polynomials of degree $2 s-1$ (on a mesh of width $h$ ). Thus if $s=2$ (i.e., piecewise cubics) then the error is of order $O\left(k^{2}\right)$ provided $k \geqq C h^{3 / 2}$. We should remark that the methods of [8] require the approximants to satisfy boundary conditions.

The methods of [10] yield error estimates of the form

$$
\left\{\int_{0}^{T}\|e(\cdot, t)\|^{2} d t\right\}^{1 / 2}=O\left(h^{2 s}+k^{*}\right)
$$

where $u \in H^{2 s, 8}(Q)$ (cf. [10]) and the admissible approximants consist of a certain class of splines which are piecewise polynomials of degree $2 s-1$ in the space variables (on a mesh of width $h$ ) and of degree $s-1$ in $t$ (on a mesh of width $k$ ). Thus for $s=2$ and $k \geqq C h^{2}$ the error is of order $O\left(k^{2}\right)$. We remark that the methods of [10] yield arbitrarily high order of accuracy (for sufficiently regular $u$ ) without requiring the admissible approximants to satisfy boundary conditions.

Acknowledgement. The author gratefully acknowledges the referee's many helpful comments and suggestions for the improvement of this paper.

Department of Mathematics

University of Cincinnati

Cincinnati, Ohio 45221 
1. S. Agmon, Lectures on Elliptic Boundary Value Problems, Van Nostrand Math. Studies, no. 2, Van Nostrand, Princeton, N. J., 1965. MR 31 \#2504.

2. I. BABUŠKA, Approximation by Hill Functions, University of Maryland Technical Note BN-648, 1970.

3. J. H. Bramble \& A. H. Schatz, "Rayleigh-Ritz-Galerkin methods for Dirichlet's problem using subspaces without boundary conditions," Comm. Pure Appl. Math., v. 23, 1970 , pp. 653-675. MR 42 \#2690.

4. J. H. Bramble \& S. R. Hilbert, "Bounds for a class of linear functionals with applications to Hermite interpolation," Numer. Math., v. 16, 1970, pp. 362-369. MR 44 \#7704.

5. J. H. Bramble \& S. R. Hilbert, "Estimation of linear functionals on Sobolev spaces with applications to Fourier transforms and spline interpolation," SIAM J. Numer. Anal., v. 7, 1970, pp. 112-124. MR 41 \#7819.

6. J. H. Bramble \& V. Thomée, "Semidiscrete least-squares methods for a parabolic boundary value problem," Math. Comp., v. 26, 1972, pp. 633-648.

7. J. H. BRamble \& M. Zlamal, "Triangular elements in the finite element method," Math. Comp., v. 24, 1970, pp. 809-820. MR 43 \#8250.

8. J. Douglas \& T. DuPONT, "Galerkin methods for parabolic equations," SIAM J. Numer. Anal., v. 7, 1970, pp. 575-626. MR 43 \#2863.

9. S. HilberT, Numerical Methods for Elliptic Boundary Value Problems, Doctoral Thesis, University of Maryland, College Park, Md., 1969.

10. J. T. KING, "The approximate solution of parabolic initial-boundary value problems by weighted least-squares methods," SIAM J. Numer. Anal., v. 9, 1972, pp. 215-229.

11. O. A. Ladyženskaja, V. A. SolonNikov \& N. N. URAL'CEVA, Linear and Quasilinear Equations of Parabolic Type, "Nauka", Moscow, 1967; English transl., Transl. Math. Monographs, vol. 23, Amer. Math. Soc., Providence, R.I., 1968. MR 39 \#3159a, b.

12. H. S. PRICE \& R. S. VARgA, Error Bounds for Semidiscrete Approximations of Parabolic Problems with Applications to Petroleum Reservoir Mechanics, SIAM-AMS Proc., vol. II, Amer. Math. Soc., Providence, R.I., 1970, pp. 74-94. MR 42 \#1358.

13. M. H. Schultz, "Approximation theory of multivariate spline functions in Sobolev spaces," SIAM J. Numer. Anal., v. 6, 1969, pp. 570-582. MR 41 \#7823.

14. B. Swartz \& B. Wendroff, "Generalized finite difference schemes," Math. Comp., v. 23, 1969, pp. 37-49. MR 39 \#1125. 